\title{
Microscopia Confocale a Fluorescenza nella localizzazione di Progenitori Staminali Renali
}

\section{Lombardi1 ${ }^{1}$ A. Sabatini ${ }^{2}$}

${ }^{1} \mathrm{CdL}$ in Biotecnologie indirizzo medico-diagnostico, Università degli Studi di Firenze; tirocinante del Laboratorio Interdipartimentale di Nefrologia Cellulare e Molecolare diretto dalla dott. P. Romagnani, Facoltà di Medicina e Chirurgia, Università degli Studi di Firenze.

2 S Labs - Riva Paradis, Switzerland as@alum.mit.edu

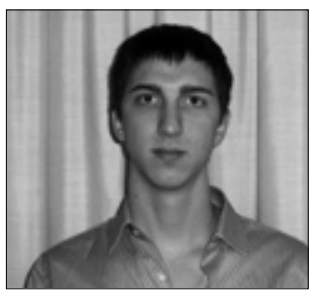

Duccio Lombardi

\section{Introduzione}

In questo breve contributo gli Autori si propongono di illustrare le principali caratteristiche della microscopia confocale a fluorescenza, metodica il cui utilizzo nell'ambito della ricerca biomolecolare in Nefrologia ha permesso la localizzazione di una popolazione con caratteristiche di staminalità nel glomerulo. Nello studio qui citato, condotto dal gruppo della Professoressa Paola Romagnani (1), le analisi al microscopio confocale di sezioni tissutali di rene adulto umano hanno rivelato la coespressione dei marker di staminalità CD24 e CD133 in cellule parietali epiteliali (PEC) localizzate a livello della giunzione tubulo-glomerulare presso il polo urinario della Capsula di Bowman. Dalla ricerca operata dallo stesso gruppo (2), è anche emerso che gli stessi progenitori nel rene embrionale condividono l'espressione del CD24 e del CD133. Tali cellule, che costituiscono i primi abbozzi del nefrone primordiale, sono destinate a scomparire durante lo sviluppo nefronico rimanendo selettivamente localizzate presso il polo urinario della Capsula di Bowman; a questo è anche associata una progressiva diminuzione della loro presenza durante l'accrescimento fetale sino ad arrivare a percentuali ancora inferiori e comprese tra il 2,0-0,5\% di tutte le cellule presenti nel rene adulto. La causa di tale riduzione risiede nel differenziamento cellulare che si verifica durante lo sviluppo renale: i vari pool cellulari passano a uno stadio terminalmente differenziato dando luogo alle varie componenti e strutture che formano il rene. A questo evento si associa però la perdita di caratteristiche proprie delle cellule staminali, quali autorigenerazione, automantenimento, multipotenza differenziativa e ampia capacità proliferativa, nonché di tali due marker. Le suddette osservazioni sono state rese possibili sia mediante citometria a flusso, già precedentemente trattata in questa rubrica (3), sia tramite microscopia confocale a fluorescenza, la quale ha, in particolare, permesso la localizzazione di queste cellule a livello tissutale. Appare quindi evidente la notevole importanza che tali due metodiche possono assumere negli studi di medicina biomolecolare.

\section{La microscopia a fluorescenza}

La capacità degli anticorpi di legare in modo estremamente specifico gli antigeni verso cui sono diretti, permette il loro utilizzo al fine di identificare determinate molecole, non solo in sezioni di tessuto e in fluidi biologici, ma anche all'interno della cellula e sulla membrana cellulare stessa. Gli anticorpi usati nella microscopia a fluorescenza sono marcati con fluorofori, ossia molecole che emettono nello spettro del visibile se eccitate con determinate lunghezze d'onda, generalmente fornite mediante laser. L'emissione del fluoroforo rende quindi possibile la localizzazione spaziale degli antigeni ricercati, cui sono legati gli anticorpi.

Il laser media il trasferimento dell'energia di eccitazione 
al campione sotto forma di "luce d'eccitamento", la quale è monocromatica, di alto livello energetico e polarizzata. Il fluoroforo eccitato da tale fonte luminosa di determinata lunghezza d'onda, ri-emette con lunghezze d'onda che variano, in base al fluoroforo scelto, e che avranno un'ampiezza maggiore, ossia una minore energia rispetto a quella fornita tramite laser (4). Da qui deriva la necessità di separare la luce eccitante, da quella emessa per fluorescenza: maggiore sarà, difatti, la quantità di sola luce ri-emessa, che il microscopio riesce a rilevare, tanto più sarà resa dettagliata l'immagine del campione. Lo schema generale di un microscopio a fluorescenza (Fig. 1) prevede che, in primis, la luce d'eccitamento sia isolata mediante l'uso di un filtro d'eccitazione, e in seguito sia indirizzata al campione. La fluorescenza emessa dal preparato passa quindi attraverso il filtro di emissione, il quale blocca tutte le lunghezze d'onda che l'operatore sceglie di eliminare in base al tipo di fluoroforo preso in considerazione nel protocollo.

Nel caso siano utilizzati più fluorofori, affinché ognuno di questi possa essere visualizzato senza la contaminazione dell'emissione degli altri fluorofori, i microscopi a fluorescenza prevedono varie unità filtranti sia di eccitazione che di emissione, ognuna delle quali è ottimizzata per un dato tipo di fluoroforo.

Il limite della semplice microscopia a fluorescenza risie-

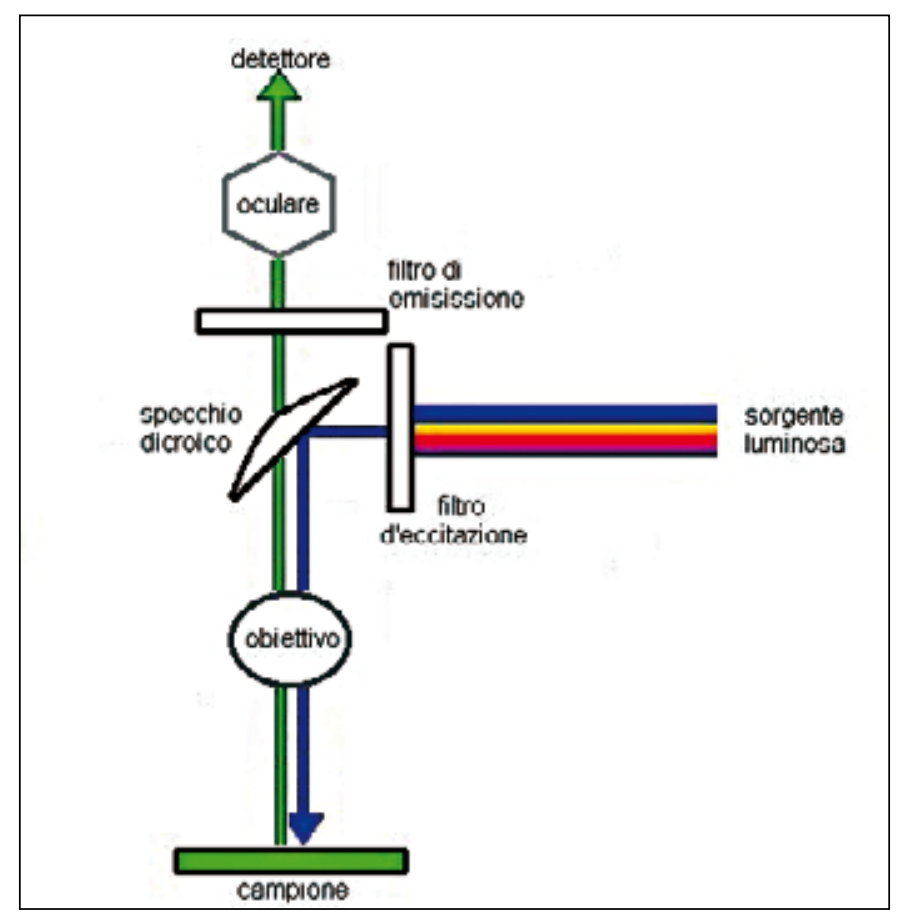

Fig. 1 - Schema generale di un microscopio a fluorescenza semplice, in cui la sorgente luminosa può essere rappresentata sia da una lampada che da un laser. de però nel rumore di fondo generato dall'illuminazione totale del campione: tutto difatti fluoresce contemporaneamente a causa dell'ampio range d'azione della luce d'eccitazione.

Al contrario un microscopio confocale a fluorescenza (Fig. 2) consta di un sistema di lenti che focalizzano la luce dal punto focale di una lente sino a un altro ben determinato punto, il quale viene a coincidere con il pinhole, permettendo di visualizzare solo tale punto focale. Ciò è reso possibile mediante l'uso di uno schermo con un piccolo foro, chiamato appunto pinhole, posto dall'altra parte del sistema di lenti. Tutta la luce derivante dal punto di focalizzazione sarà convogliata presso il pinhole, eliminando invece tutte le fonti luminose derivanti da altre direzioni.

Questo sistema fa si che solo un determinato punto del campione emetta e sia quindi reso visibile. In questo modo si ha il vantaggio di poter analizzare un singolo piano di focalizzazione alla volta, escludendo tutti quelli non desiderati.

Nella microscopia confocale il laser è riflesso su di uno specchio dicroico (Fig. 2) e colpisce poi altri due specchi, montati su motori, che indirizzano il laser attraverso il campione. Si ha a questo punto emissione da parte dei fluorofori, con la luce emessa che sarà scandita da-

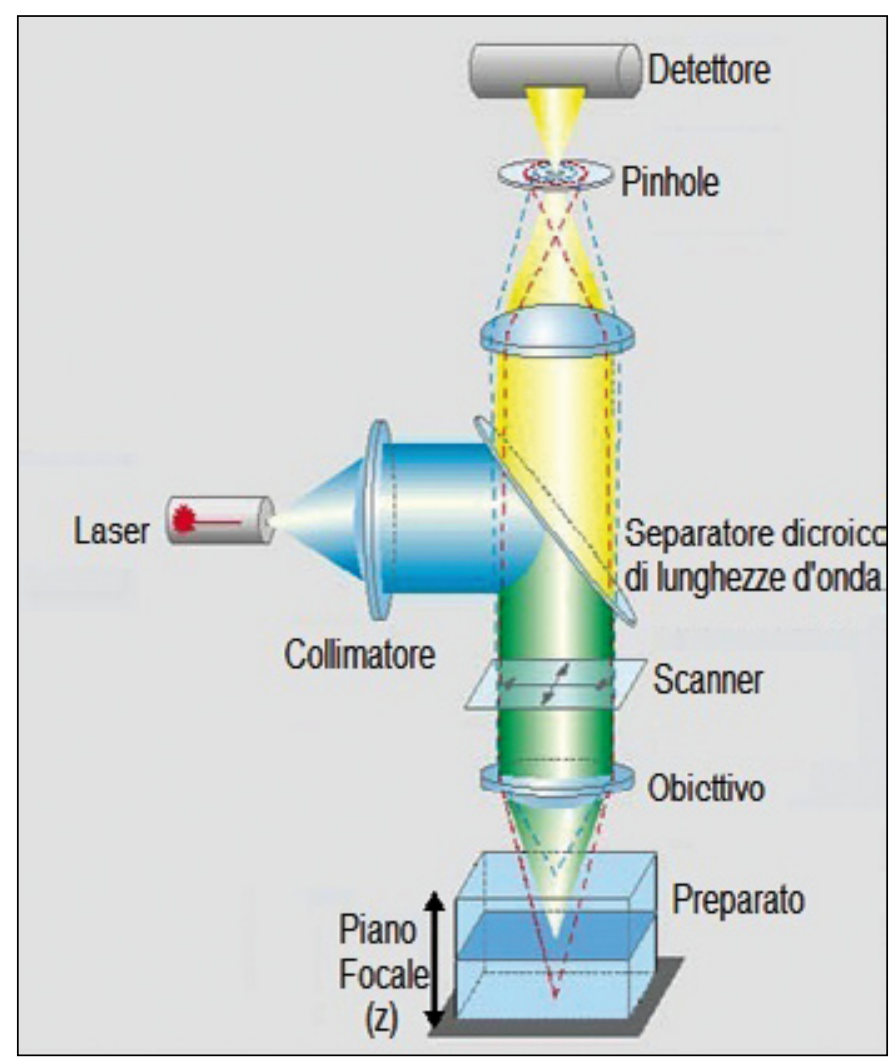

Fig. 2 - Riproduzione schematica di un microscopio confocale a fluorescenza, di cui sono evidenziati i componenti fondamentali (7). 
gli stessi specchi usati nel caso della luce d'eccitazione. La luce emessa è dicroizzata e focalizzata a livello del pinhole, ove sono effettuate misurazioni da detector che ricostruiscono l'immagine su di un computer, un pixel per volta (6).

La ricostruzione dell'immagine al computer permette non solo di localizzare un determinato antigene, in un tessuto, grazie alla colorazione cui è associato l'anticorpo che lo ha legato, ma al contempo rende evidente un'eventuale co-localizzazione, e quindi coespressione, di due marker. Nonostante i diversi fluorofori emettano in bande che sono separate (diversa colorazione), il software leggendo il segnale di entrambi nello stesso punto renderà tale segnale come una semplice addizione dei colori. Sarà quindi visualizzato, ad esempio, un segnale giallo qualora in quel punto vi fossero il verde di un marker e il rosso dell'altro marker. Ciò assume una particolare rilevanza nel caso di immunofluorescenze con double staining, analisi che nello studio qui esaminato (1) ha permesso la localizzazione della popolazione di cellule progenitrici renali a livello della capsula di Bowman.

\section{Preparazione del campione}

Lo studio in oggetto (1), ha messo in evidenza la presenza, a livello renale, di una popolazione di progenitori, contraddistinta dall'espressione dei marcatori di membrana CD24 (marker qualificante progenitori renali embrionali) e CD133 (espresso selettivamente da cellule staminali adulte). Questa caratterizzazione da un punto di vista pratico prevede la ricerca di due antigeni contemporaneamente, processo chiamato "double staining". Per "double staining" si intende una doppia colorazione, costituita da due singole colorazioni consecutive, e generalmente attuata mediante immunofluorescenza indiretta. Sono quindi usati un anticorpo primario per il primo antigene (CD24) e un anticorpo secondario coniugato a un fluoroforo al fine d'individuare tale anticorpo primario. In una fase immediatamente successiva sarà marcato un diverso antigene (CD133) con un secondo anticorpo primario il quale è poi rilevato da un secondo anticorpo secondario, coniugato a un diverso fluoroforo. I due anticorpi primari devono essere di due diversi isotipi (per esempio, IgG1 e IgG2b), questo affinché gli anticorpi secondari possano distinguere tra questi due primari. Inoltre l'anticorpo secondario dovrà essere ottenuto da una specie diversa rispetto a quella da cui si ottiene il primario: se, ad esempio, usiamo un anticorpo primario ottenuto nel topo, dovremo usare un anticorpo secondario ottenuto nella capra o comunque sia in una specie diversa da quella di topo.
Deve essere posta attenzione, in particolar modo, all'ordine con cui sono marcati gli antigeni. Gli antigeni di membrana, devono essere marcati per primi, passando successivamente a quelli citosolici, i quali richiedono la permeabilizzazione della membrana stessa.

Nella preparazione del campione è importante che questo non vada mai "a secco" e che, effettuato un primo lavaggio delle sezioni di tessuto fissate su vetrino, sia attuata un'opportuna fissazione delle cellule o del tessuto. Questo permette di mantenere la distribuzione degli antigeni e di preservare la morfologia cellulare evitando artefatti: la cellula apparirà esattamente com'era al momento della fissazione.

Le sezioni di tessuto sono quindi coperte con il Blocking Serum, il quale neutralizza ogni possibile sito del campione che gli anticorpi secondari potrebbero legare: l'anticorpo secondario legherà e rivelerà così solo l'anticorpo primario per cui è specifico.

$\mathrm{E}$ eseguito un successivo lavaggio ed è quindi possibile marcare per immunofluorescenza indiretta incubando con un primo anticorpo diretto verso l'antigene d'interesse. Un successivo lavaggio elimina l'eccesso di anticorpo primario legato su siti aspecifici, e l'anticorpo primario è poi localizzato mediante l'uso dell'anticorpo secondario, attuando un'incubazione in un luogo cui si deve avere totale assenza di luce. $\grave{E}$ in questo modo evitato il "photobleaching", ossia l'alterazione e la perdita d'intensità di fluorescenza del fluoroforo coniugato all'anticorpo secondario, evento che porterebbe verso segnali più deboli. In fine è rimosso mediante lavaggio anche l'eccesso di anticorpo secondario.

L'intera operazione è poi ripetuta per l'individuazione del secondo marker di superficie, con la sola differenza che il processo di marcatura riprende dal passaggio del Blocking Serum: non si necessita ossia di un secondo fixing cellulare.

Lo step finale prevede il montaggio con vetrino coprioggetto, la rimozione di eventuali bolle d'aria e la visualizzazione mediante microscopia del preparato.

Le immagini riportate in questo contributo fanno riferimento a un'immunofluorescenza diversa da quella effettuata e pubblicata nello studio (1), ma riprodotta dagli Autori appositamente per questo articolo, su sezioni tissutali di $5 \mu \mathrm{m}$ di rene umano adulto, fissate in acetone. In particolare il segnale verde è associato all'anticorpo diretto contro il CD133, mentre la colorazione rossa mostra la presenza del CD24. Per la colorazione del nucleo è utilizzato il TO-PRO-3 (blu).

La sovrapposizione dei colori associati ai due relativi anticorpi (verde + rosso), appare nelle immagini di microscopia confocale di colore giallo. Questi sono i siti dove il CD133 ed il CD24 sono coespressi. 


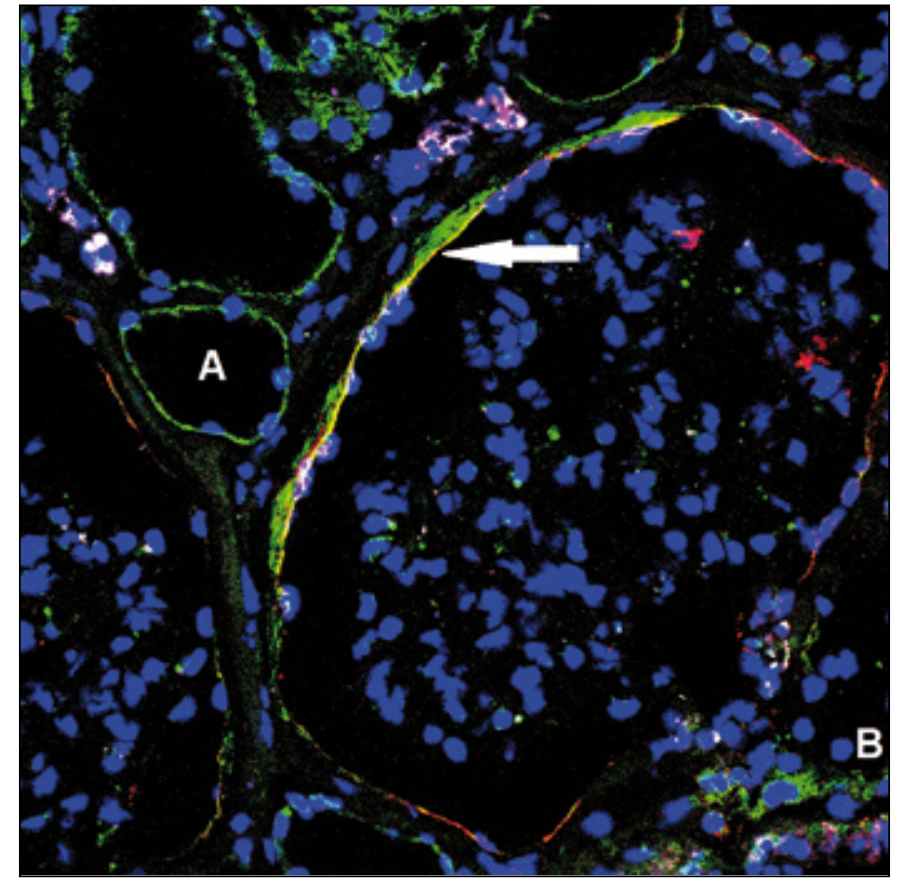

Fig. 3 - Capsula di Bowman (freccia) in cui è possibile notare una coespressione dei marker CD24 (rosso) e CD133 (verde), resa evidente dalla colorazione gialla localizzata presso il polo urinario. Il polo urinario è caratterizzato dalla presenza di strutture tubulari (A) individuate dal solo CD133. Presso il polo vascolare, distinguibile grazie a parte della struttura vascolare $(B)$, è invece possibile notare come sia assente la coespressione di tali due marker. Il blu identifica nuclei cellulari.

Queste cellule e localizzate al polo urinario della Capsula di Bowman esprimono anche altri marcatori distintivi di staminalità, quali Nanong ed Oct4. Tale pool cellulare, con evidenti caratteristiche di staminalità, appare quindi fornito dei requisiti base per dar luogo alla rigenerazione tissutale di alcune parti del rene adulto. Questo è stato dimostrato, sempre ad opera del gruppo di ricerca della Professoressa Paola Romagnani, mediante iniezioni di queste cellule in topi cui era stata indotta nefropatia, e in cui è stato possibile osservare una diminuzione di proteinuria e dei livelli di azoto ureico, rispetto ai controlli negativi che non ricevevano tali cellule CD24+ CD133+ (1). Questo aspetto, e le relative possibilità legate all'utilizzo di tali cellule in vivo, sarà in particolare analizzato e approfondito nel prossimo numero di questa rubrica.

\section{Indirizzo degli Autori:}

Duccio Lombardi

Via B. Scala 23

50126 Firenze

lombarduccio@alice.it

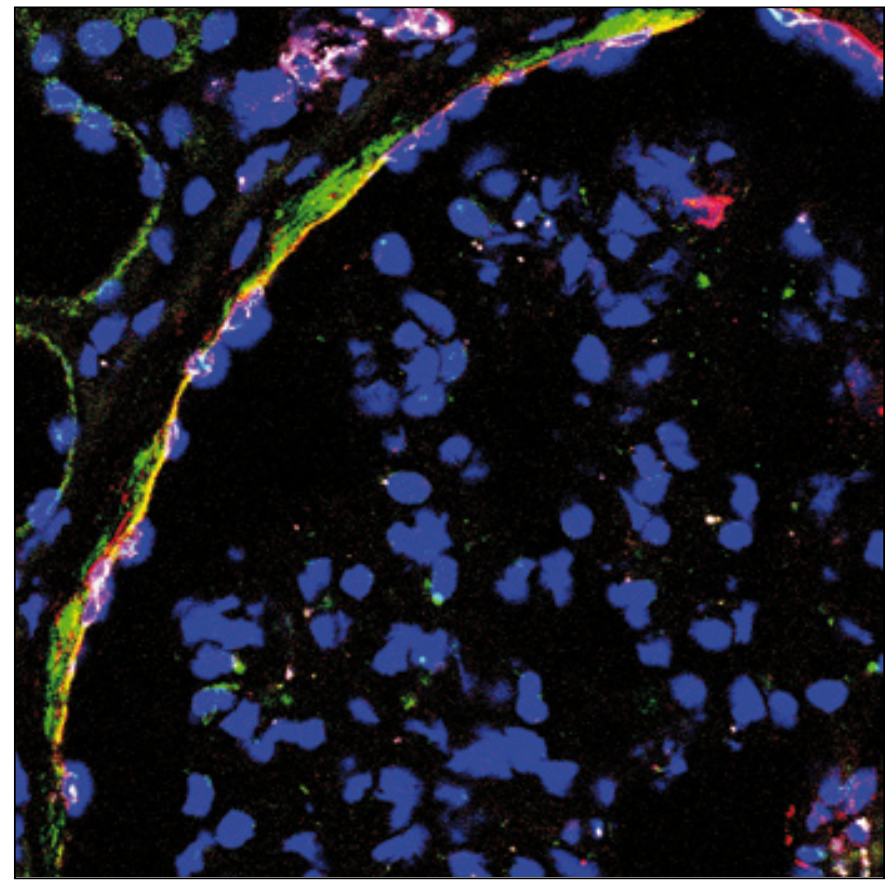

Fig. 4 - Particolare del polo urinario della stessa struttura capsulare.

\section{Bibliografia}

1. Sagrinati C, Netti GS, Mazzinghi B, et al. Isolation and characterization of multipotent progenitor cells from the Bowman's capsule of adult human kidneys. J Am Soc Nephrol 2006; 17: 2443-56.

2. Lazzeri E, Crescioli C, Ronconi E, et al. Regenerative potential of embryonic renal multipotent progenitors in acute renal failure. J Am Soc Nephrol 2007; 18: 3128-38.

3. Lombardi D. Individuazione di una popolazione staminale renale: Bio-tecniche e Bio-tecnologie. Giornale di Tecniche Nefrologiche \& Dialitiche 2010; 22(1).

4. Herman B. 2002, Fluorescence Microscopy, Current Protocols in Immunology, 21.2.1-21.2.10.

5. Ottenuto da http://www.laboderisio.it/i/IMMUNOFLUORESCENZA.pdf

6. Prasad V, et al. Confocal Microscopy of Colloids. J. Phys.: Cond. Mat. 19, 113102, 2007

7. Ottenuto da http://www2.bio.ku.dk/microbiology/research/clsm.asp

Le immagini che illustrano l'articolo sono pubblicate a colori sul sito www.sin-italy.org 